\title{
Evaluation Abilities and Requirements of the Wind Tunnel Hardware-in-the-loop Simulation for Evaluation of Missiles' Flight Control Systems
}

\author{
Xing-bao Yang ${ }^{a}$ and Min Huang \\ Dalian Naval Academy, Dalian, Liaoning, 116018, China
}

\begin{abstract}
In order to determine the flight control system (FCS) performances that wind tunnel hardware-in-the-loop simulation (WT-HILS) can evaluate, and lay foundations for designing the WT-HILS evaluation methods, the evaluation abilities and requirements of WT-HILS for FCS evaluation were analyzed. Firstly, the features of WT-HILS were pointed out. Then, according to the features of WT-HILS, what FCS performances WT-HILS can evaluate were analyzed, and the differences of evaluation abilities among the WT-HILS, the traditional hardware-in-the-loop simulation (HILS), and flight tests were compared. Lastly, from the aspects of testing, data processing, and FCS performance determination, the specific requirements of WT-HILS for evaluating FCS were analyzed.
\end{abstract}

\section{NOMENCLATURE}

$\varphi, \varphi_{i}, \varphi_{o}, \varphi_{m}, \varphi_{e}$ : Pitch angle, pitch angle input command, pitch angle output, maximum value of the output pitch angle, steady-state value of the output pitch angle

$e_{t r}$ : Steady-state error which is defined as the difference between $\varphi_{i}$ and $\varphi_{e}$

$t_{s}$ : Transient time, defined as the time required for the output pitch angle reaching to $98 \%$ or $102 \%$ of the $\varphi_{e}$

$\sigma$ : Overshoot, defined as the difference between $\varphi_{m}$ and $\varphi_{e}$

\section{Introduction}

Wind-tunnel hardware-in-the-loop simulation [1-2] was proposed aiming at improving the reality of the traditional hardware-in-the-loop simulation. By putting the aerodynamic objects in the wind tunnel airflow to perform rotational motions under the action of controllers, the aerodynamics and rotational motions can be reproduced realistically in WT-HILS. Compared to the traditional HILS using mathematical models to model the aerodynamics and motions, WT-HILS is closer to flight tests and can give more reliable FCS evaluation results. Based on this, many departments are developing such a

\footnotetext{
${ }^{\text {a }}$ Corresponding author : yxbjtxy@163.com
} 
technique in an attempt to apply WT-HILS to evaluate FCS. Among these departments, some uses WT-HILS to evaluate the flight control laws with the method of rapid control prototyping (RCP) so as to early detect the FCS problems before the real FCS hardware is developed, such as the University of Cambridge [3] and the University of Bristol [4] in UK, the SibNIA [5] in Russia, the University of Texas [6] in USA, the China Academy of Aerospace Aerodynamics (CAAA) [7] and the China Aerodynamics Research and development center (CARDC) [8-10] in China. Some attempts to use WT-HILS to evaluate the hardware integrated FCS before flight tests and after HILS so as to detect those FCS problems that may occur in flight tests and are not detected in HILS and reduce the risks of flight tests, such as the Arnold Engineering Development Center (AEDC) [11] and the Physical Sciences Incorporation (PSI) [12] in USA.

The above researches mainly concentrated on the development of testing rigs and few researched the WT-HILS evaluation methods. However, the FCS evaluation methods are the key technology needed to be solved for applying WT-HILS to evaluate FCS [13-16]. Before designing the WT-HILS evaluation methods, what FCS performances can be evaluated by WT-HILS should be first known and specific requirements of the WT-HILS evaluation methods should be listed so as to indicate directions for the design of WT-HILS evaluation methods. Therefore, the evaluation abilities and the requirements of WT-HILS for the evaluation of missiles' FCSs were analysed in this paper.

\section{WT-HILS features}

Before researching the WT-HILS technique, features of WT-HILS should be first pointed out so as to propose practicable WT-HILS evaluation abilities and requirements of the WT-HILS evaluation methods. As the WT-HILS is based on wind tunnels, it has the following three obvious features:

(1) In WT-HILS, translational motions or the flight trajectory of the aircraft cannot be reproduced in wind tunnels during one test. In current wind tunnels, the support rigs can enable rapid change of the model positions. However, the space of the wind tunnel test section is limited, which makes the translational motions of the aircraft test articles are limited and the aircrafts cannot fly the whole flight trajectory as in real flights. In order to model the translational motions by WT-HILS, a common method is to calculate the translational motions in real-time with the measured data from wind tunnels.

(2) In WT-HILS, the amplitude of the aircraft speed cannot be changed in real time as in real flights during one test. During flight tests, the amplitude of aircraft speed may change rapidly. While in WT-HILS, the wind tunnel airflow speed cannot change as quickly as in real flights during one test at present. Besides, current wind tunnels are classified into wind tunnels with various range of speed, such as the low-speed wind tunnels, the transonic wind tunnels, the supersonic wind tunnels. There is not a wind tunnel that can generate an airflow that can vary from low speed to supersonic speed or even hypersonic speed. Therefore, it is quite hard for WT-HILS to reproduce the motions with speed change in real time at present. The FCS performance concerning speed changes will not be able to be evaluated by WT-HILS.

(3) In WT-HILS, the direction of the aircraft speed or the velocity directional angles of the aircraft cannot be changed in real time as in real flights during one test. In real flights, the attitude angles (the pitch angle and the yaw angle) consist of not only the aerodynamic angles but also the velocity directional angles. As the aerodynamic angles (the angle of attack and the angle of sideslip) are driven by the wind tunnel support rigs, aerodynamic angles can be reproduced in real time in the WT-HILS by moving the rig in real time or letting the aircraft freely rotate on the rig. While in current wind tunnels the wind tunnel airflow direction cannot change or cannot change as quickly as the change of the aircraft velocity direction in real flights, the aircraft velocity directions cannot be changed in real time in WT-HILS. To model the real attitude motions in real time, the velocity directional angles should be calculated in real time with the wind tunnel balance measured aerodynamic forces.

\section{Evaluation abilities of WT-HILS}




\subsection{What FCS performances WT-HILS can evaluate?}

Generally, a missile's FCS performances can be divided into the navigation/guidance performances, attitude stability and control performances. The navigation/guidance performances are used for representing the FCS performances concerning both translational motions and attitude motions. The indexes of navigation/guidance performances include velocity indexes, height indexes, taking off and landing indexes, endurance indexes, guidance precision and so on [17]. The attitude stability and control performances are used for representing FCS performances concerning attitude motions. The indexes of attitude stability and control performances include attitude stability indexes, attitude control indexes, and agility indexes and so on [17-18].

As introduced in the WT-HILS features, although the translational motions, the velocity directional angles and the attitude angles cannot be reproduced as in real flights in wind tunnels, velocity directional angles, attitude angles and translational motions can be calculated in real time with the measured data from wind tunnels. However, current wind tunnels generally cannot change airflow speed as in real flights and the influence of speed change can hardly be compensated by numerical methods, because once the airflow speed cannot be adjusted in real-time, the different airspeeds' aerodynamic loadings for making up the influence of airspeed change cannot be obtained. Therefore, before the wind tunnel airflow can be adjusted in real-time, the FCS performance involved with speed changes cannot be evaluated by WT-HILS. The following is a brief description of what FCS performance WT-HILS can evaluate.

(1) Navigation/guidance performances under steady flights. As in steady flights the translational accelerations are 0 , navigation/guidance performances under such flight conditions can be evaluated by WT-HILS, such as the target tracking indexes under steady flights, the hovering radius and cycle indexes under steady hovers. While due to the limitation of speed change, the velocity indexes, taking off and landing indexes, guidance precision cannot be evaluated.

(2) Attitude stability performances. The WT-HILS can evaluate the stability performances under various kinds of attitudes, whether it is the normal motion or the unsteady motion. Besides, an important evaluation ability of WT-HILS is to evaluate the stability boundaries of FCS, which can also be called the FCS control limitations. When the aircraft's attitudes exceed the stability boundaries, the FCS will become invalid. Therefore, by obtaining the stability boundaries with WT-HILS, those manoeuvres out of the attitude stability boundaries can be avoided to perform in flight tests and the risks of flight tests will be reduced.

(3) Attitude control performances under normal motions. Under normal motion conditions (motions with small amplitude), the aircraft motions can be decoupled into longitudinal motions and lateral-directional motions. The longitudinal motions include the short-term mode and the long-term mode. The short-term mode is mainly relative to the angle of attack and the pitch angle, while the influence of speed change is small and can be neglected. The long-term mode is mainly relative to the velocity and flight path angle. The lateral-directional motions include the Dutch roll mode, roll mode and spiral mode. These modes are mainly relative to the roll angle, angle of sideslip, yaw angle, while the influence of speed change is usually small and can be neglected. Therefore, attitude control performances under longitudinal short-term motions and lateral-directional motions can be evaluated by WT-HILS, while those under longitudinal long-term motions cannot be evaluated by WT-HILS.

(4) Attitude control performances under unsteady manoeuvres. The unsteady manoeuvres are mainly relative to large angle of attack or large angle of sideslip, and have quite obvious nonlinear aerodynamic characteristics, which will result in strong couplings between aerodynamics and motions. To modelling these manoeuvres realistically, the WT-HILS rigs should permit free rotations or free manoeuvres so as to avoid the calculating of aerodynamic angles with measured moments and the aerodynamics and motions can counteract with each other as that of real flights. In this way, FCS performances under unsteady manoeuvres can be effectively evaluated by WT-HILS, while it is quite difficult for HILS to evaluate such performances. Some departments had developed rigs to evaluate the control algorithms of limit cycles [4], the free spin [5] and the pitching-rolling coupling motions [9]. 
(5) Heading agility indexes. Aircraft agility can be divided into the flight path agility and the heading agility. The flight path agility means "the ability to change the direction and amplitude of velocity with FCS". Apparently, it cannot be evaluated by WT-HILS. The heading agility means "the ability to change the head directions (such as pitch and yaw) with FCS". As the heading agility is mainly relative to the attitude motions and the manoeuvring time, it can be evaluated by WT-HILS.

Several sample indexes that WT-HILS can evaluate are listed in the Table 1. These indexes are used to represent the attitude tracking performance of the missile's pitching control system (shown in Figure 1), which belongs to the attitude control performances under normal motions.

Table 1. Sample indexes that WT-HILS can evaluate.

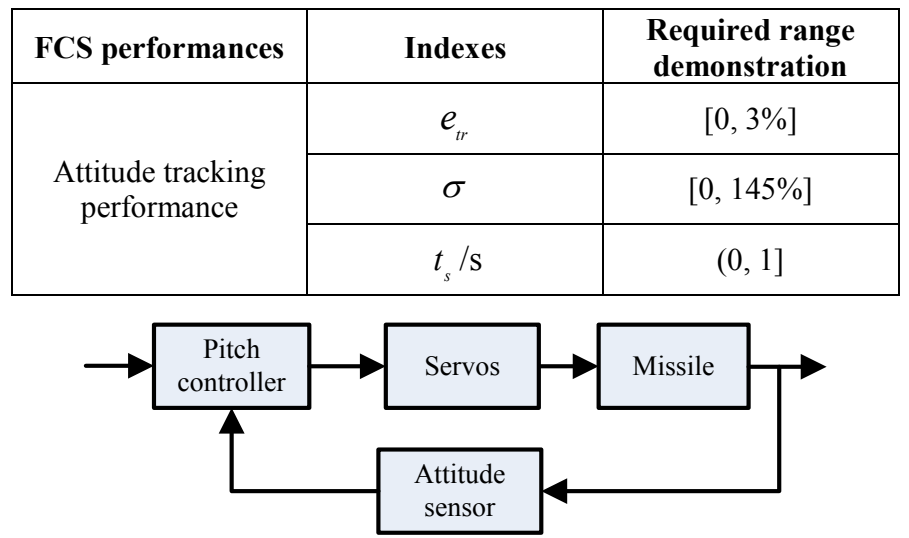

Figure 1. The missile's pitching control system.

\subsection{Comparisons of the evaluation abilities among WT-HILS, HILS, flight tests}

As analyzed above, WT-HILS cannot evaluate all indexes of FCS. The evaluation abilities among WT-HILS, HILS and flight tests are compared as follows.

\subsubsection{Comparisons of the evaluation abilities between WT-HILS and HILS}

By putting the hardware integrated aircraft in the wind tunnel airflow, WT-HILS can evaluate the FCS more realistically than HILS and can evaluate some FCS indexes that are difficult to be evaluated in HILS. Though less realistic than WT-HILS, some FCS performances can only be evaluated by HILS on ground.

(1) The WT-HILS can evaluate the navigation/guidance performances under steady flights, attitude stability and control performances under longitudinal short-term motions and lateraldirectional motions, and heading agility indexes more realistically than HILS.

(2) The WT-HILS can evaluate attitude stability and control performances under unsteady maneuvers accurately which can hardly be evaluated by HILS because it is quite difficult for HILS to model unsteady aerodynamics and motions accurately.

(3) HILS can evaluate those FCS performances related to velocity change, such as the velocity indexes, taking off and landing indexes, guidance precision, and longitudinal long-term performances, while these cannot be evaluated by WT-HILS at present.

(4) HILS can evaluate the robust performances of FCS by performing a series of simulation tests in a short time with many kinds of combined disturbances added to various system parameters. While the robust performances of FCS can hardly be evaluated by WT-HILS because on the one hand, the disturbances, such as the center of gravity disturbance, the moment of inertia disturbance, and the wind disturbances are hard to add into the WT-HILS, on the other hand, it is difficult for WT-HILS to perform a lot of wind tunnel tests due to the high cost and long period of wind tunnel tests. 


\subsubsection{Comparisons of the evaluation abilities between WT-HILS and flight tests}

(1) WT-HILS can evaluate FCS performances with almost zero risks only if the aircraft test article protection measures are taken. Therefore, the aircraft test article can be totally released so as to test the control limitations of FCS while it is of high risks and costs to do so in flight tests.

(2) Though WT-HILS is less risky, it cannot replace flight tests. On the one hand, the test environment in flight tests is completely real and the FCS evaluation results of flight tests are absolutely reliable. On the other hand, many FCS performances relating to the speed change cannot be evaluated by WT-HILS while flight tests can evaluate all the attitude and guidance control abilities during the whole flight trajectory.

\section{Requirements of the WT-HILS evaluation methods}

\subsection{The technical content of the WT-HILS evaluation methods}

The technical content of a WT-HILS evaluation method can be generally divided into three steps (shown in Figure 2): 1) aiming at the evaluated FCS indexes, design the WT-HILS test method, which should definite the test system scheme, and the test performing scheme (including the test types, test inputs and test procedures); 2) design the data processing method, which should transfer the measured data to the indexes; 3) design the performance determination method, which should give the index level criterions so as to determine the index levels and judge whether the indexes are satisfied or have enough performance levels.

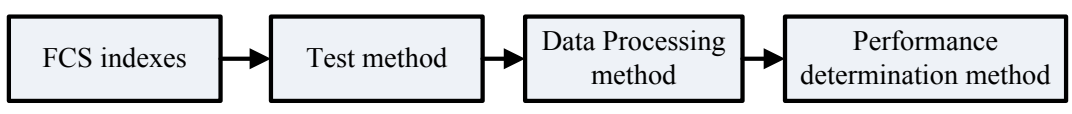

Figure 2. Procedures of a WT-HILS evaluation method.

\subsection{Requirements of the WT-HILS evaluation methods}

For the convenience of analysis, a missile's guidance control system (shown in Figure 3 ) is taken as the sample evaluation object here. The requirements of the WT-HILS evaluation methods for evaluating the guidance control system are introduced as follows.

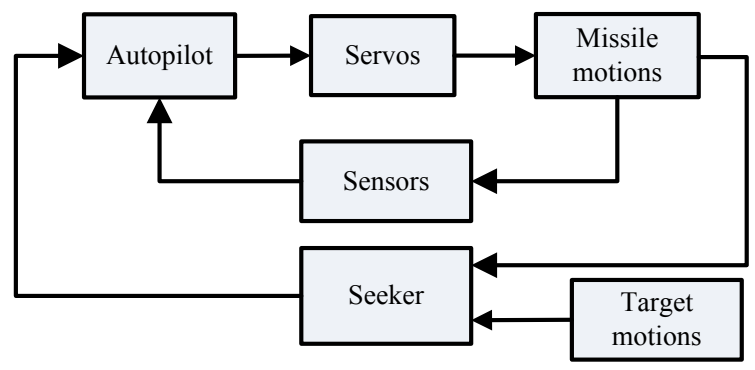

Figure 3. A missile's guidance control system.

\subsubsection{Requirements of the test method}

(1) Requirements of the WT-HILS test system. To let the wind tunnel airflow act on the real aircraft, the aircraft test article containing the FCS hardware should be put inside the wind tunnel test section. Due to the limitation of translational motions, attitude angles and translational motions should be calculated numerically with the measured aerodynamic forces and aerodynamic angles. The aerodynamic angles should be measured by the original gyro located inside the test article or other 
angular sensors, and the aerodynamic forces should be measured by an aerodynamic balance. To permit rotations of the aircraft test articles, special model support rigs are needed. These rigs are different from the traditional ones. The traditional model support rigs are usually used to ensure 1DOF free oscillations or predefined forced motions, while the WT-HILS rigs should allow 3-DOF (pitch, yaw, and roll) rotations by responding to the actions of servos. If some or all of the 3-DOF aircraft rotations need to be driven by the support rigs, aerodynamic moments should be measured by the balance in real-time to calculate the aerodynamic angle commands for the support rigs.

To provide the measuring environment for the linear accelerometer, an accelerometer simulator is needed to reproduce the linear accelerations. To provide the measuring environment for the seeker, a dark chamber containing the target simulator and turntables for simulating the missile/target relative information is needed. To ensure the time consistency of the running of real hardware and the numerical calculations, a real-time data acquisition and control system is needed to real-time collect data, process data, transmit signals and control devices (wind tunnel, model support rig, accelerometer simulator, target simulator, turntables). According to these requirements, a sample test system scheme for evaluating the missile's guidance control system is shown in Figure. 4.

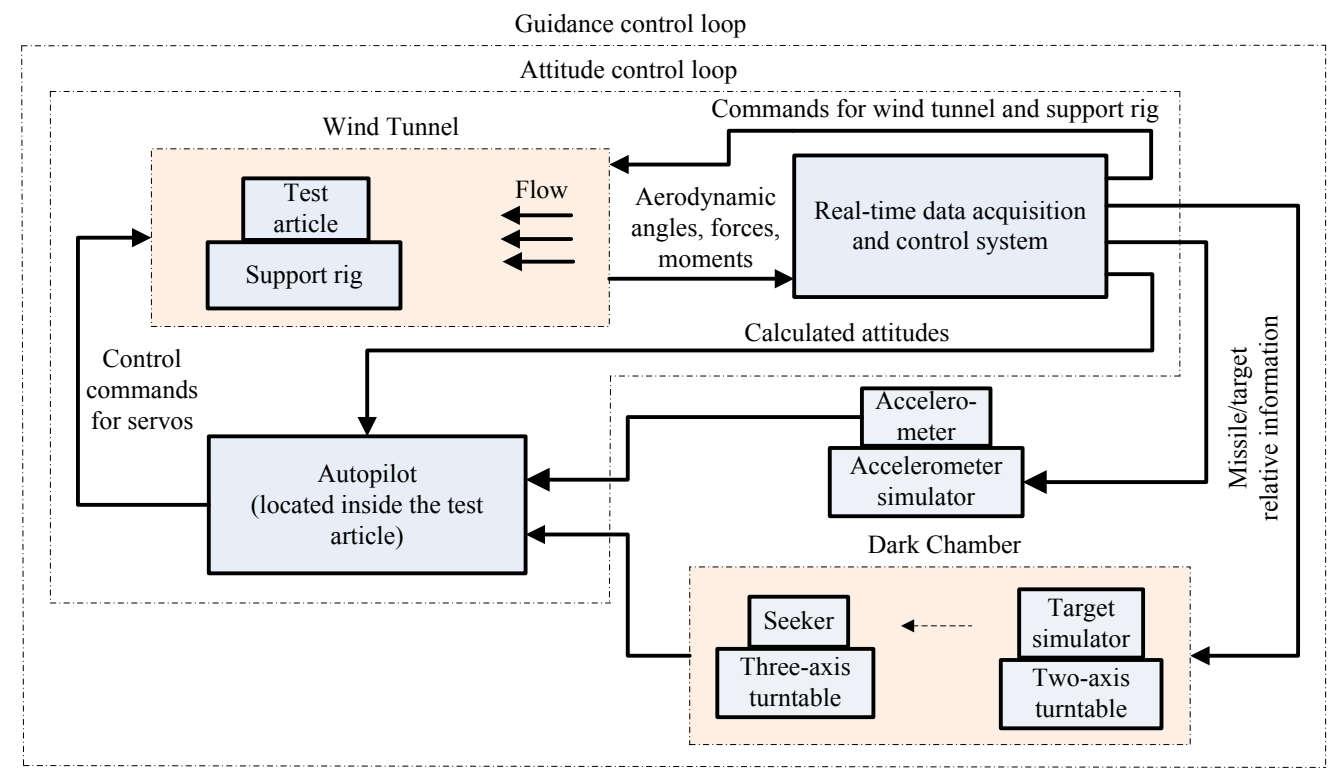

Figure 4. A sample WT-HILS system scheme for the missile's guidance control system.

(2) Requirements of the test performing. To different FCS performance indexes, tests of different types should be performed. To evaluate the attitude stability performance indexes, such as the attitude control limitation index, attitude stability tests should be obtained. The inputs for this kind of tests should be a gradually increased or gradually decreased attitude input command. To evaluate the attitude control performance indexes, such as the attitude tracking indexes in Table 1 and spin recovery indexes [5], attitude control tests should be performed. The inputs for this kind of tests should be the pre-input attitude commands first for driving the aircraft into a specific attitude condition, and then the desired attitude input commands for changing the specific attitude to the desired attitude. To evaluate the heading agility indexes, heading agility tests should be performed. The inputs for this kind of tests should be multistep attitude input commands so as to obtain the time for changing one attitude to another attitude. To evaluate the guidance performance indexes, which are the target tracking indexes for the FCS in Figure 3, guidance tests should be performed. The inputs for this kind of tests should be the target positions. Test procedures for guiding how to perform these tests should be specially designed towards different test types. For example, to evaluate the spin recovery indexes, what attitude input commands should be so as to reproduce spin motions, what desired 
attitude input commands should be so as to recover normal motions from spin motions, how to apply these commands and how to record data should be given in the test procedure [5].

\subsubsection{Requirements of the data processing method}

After the above tests, various data can be obtained. Some data can be directly used as the indexes for performance determination, such as the flight performance parameters (aerodynamic angles, velocity directional angles, attitude angles, aircraft positions, overloading), while some have to be processed to the indexes, such as the indexes Table 1. To illustrate what a data processing method should be adopted, the indexes in Table 1 are taken as an example. After an attitude control test, an attitude tracking curve (a sample curve is shown in Figure 5) can be obtained. From the curve, the $\varphi_{m}, \varphi_{e}$, and $t_{s}$ can be read. Then, the indexes $\sigma$ and $e_{t r}$ should be calculated with the following equations:

$$
\begin{gathered}
\sigma=\left|\varphi_{m}-\varphi_{e}\right| / \varphi_{e} \times 100 \% \\
e_{t r}=\left|\varphi_{e}-\varphi_{i}\right| / \varphi_{i} \times 100 \%
\end{gathered}
$$

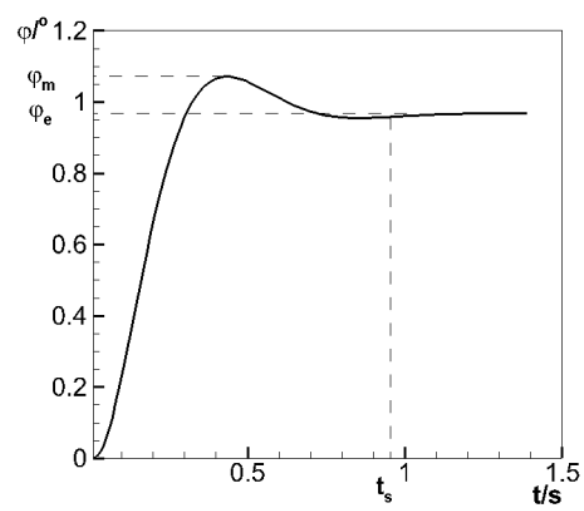

Figure 5. A sample attitude tracking curve.

\subsubsection{Requirements of the performance determination method}

After obtaining the indexes, performance determination methods are needed. These methods should give the index level criterions, which should specify different regions of performance levels allowed for the indexes. By comparing the indexes with their corresponding level regions, their performance levels can be determined, which will show how well the evaluated performances are.

Table 2. Sample level regions for the attitude tracking indexes in Table 1.

\begin{tabular}{|c|c|c|c|}
\hline \multirow{2}{*}{$\begin{array}{c}\text { Performance } \\
\text { indexes }\end{array}$} & \multicolumn{3}{|c|}{ Level dividing } \\
\cline { 2 - 4 } & $\begin{array}{c}\text { Level 1 } \\
\text { (Satisfying) }\end{array}$ & $\begin{array}{c}\text { Level 2 } \\
\text { (Acceptable) }\end{array}$ & $\begin{array}{c}\text { Level 3 } \\
\text { (Controllable) }\end{array}$ \\
\hline$e_{t r}$ & {$[0,1 \%]$} & $(1 \%, 2 \%]$ & $(2 \%, 3 \%]$ \\
\hline$t_{s} / \mathrm{s}$ & $(0,0.3]$ & $(0.3,0.6]$ & $(0.6,1]$ \\
\hline$\sigma$ & {$[0,45 \%]$} & $(45 \%, 100 \%]$ & $(100 \%, 145 \%]$ \\
\hline
\end{tabular}


The performance determination method for indexes in Table 1 is taken as an example here. The method sets the sample level regions of the attitude tracking indexes shown in Table 2. Supposing the value of $e_{t r}$ is $0.7 \%$. Comparing it with the region in Table 2 , it is in the region of level 1 . This shows the steady-state error of attitude tracking is satisfying.

\section{Conclusions}

The evaluation abilities and requirements of the WT-HILS for evaluation of FCS were analyzed and compared with the traditional HILS in this paper. The followings are summarizations.

(1) WT-HILS has three main features: limitation of aircraft translational motions in wind tunnels; limitation of wind tunnel airflow speed change; velocity directional angles (flight path angle, heading angle) cannot be reproduced in wind tunnels. Among the three features, velocity directional angles and translational motions can be numerically reconstructed in real-time with the data measured from wind tunnels, while the influence of speed change cannot be compensated numerically.

(2) Due to the limitation of airflow speed change, the WT-HILS evaluation abilities are limited: the guidance performances under steady flights, the stability and control performances under longitudinal short-term motions, lateral-directional motions and unsteady manoeuvres, and the heading agility indexes can be evaluated by WT-HILS, while the FCS performances relating to speed change, such as the guidance precision, longitudinal long-term control performances and flight path agility cannot be evaluated by WT-HILS. Then, the evaluation abilities among the WT-HILS, HILS and flight tests were compared.

(3) The technical content of a WT-HILS evaluation method includes the test method, the data processing method, and the performance determination method. According to the technical content, towards a sample missile's guidance control system, the requirements of the WT-HILS evaluation methods for this FCS were determined and examples of the test system schemes and the test performing schemes, the data processing methods, and the performance determination methods were given for illustrations.

\section{References}

1. Bacic M, Daniel R. Towards a low-cost hardware-in-the-loop simulator for free flight simulation of unmanned air vehicles. AIAA Modeling and Simulation Technologies Conference and Exhibit, AIAA 2005-6102, 2005.

2. Bacic M, Daniel R. Hardware-in-the-loop simulation of aerodynamic objects. AIAA Modeling and Simulation Technologies Conference and Exhibit, AIAA 2007-6465, 2007.

3. Papageorgiou G, Glover K. Two-degree-of-freedom control of an actively controlled wind-tunnel model. Journal of Guidance Control and Dynamics, 2002, 25(3): 510-516.

4. Richardson T S, Dubs A, Lowenberg M H, Jones C D. Wind-tunnel testing of a dynamic statefeedback gain scheduled control system. AIAA 2005-5976, 2005.

5. Sohi N P. Modeling of spin modes of supersonic aircraft in horizontal wind tunnel. 24th International Congress of the Aeronautical Sciences, 2004.

6. Gans N R, Dixon W E, Lind R, Kurdila A. A hardware in the loop simulation platform for visionbased control of unmanned air vehicles. Mechatronics, 2009, 19(2009): 1043-1056.

7. $\mathrm{Hu}$ J, Li Q. Primary investigation of the virtual flight testing techniques in wind tunnel. Journal of Experiments in Fluid Mechanics, 2010, 24(1): 95-99. (in Chinese)

8. Li H. Study on the similarity critiria and simulation method of the wind tunnel based virtual flight testing. PhD Thesis, China Aerodynamics Research and Development Center, 2012. (in Chinese)

9. Zhao Z L, Wu J Q, L H, Zhou W Q, Mao D Y, Yang H Y. Investigation of virtual flight testing technique based on $2.4 \mathrm{~m}$ transonic wind tunnel. Acta Aeronautica et Astronautica Sinica, 2016, 37(2): 504-512. (in Chinese) 
10. Nie B W, Zhu M H, Guo L L, Wen Y C, Jiang M. Key technique and design scheme of the wind tunnel virtual flight system. 2012 Chinese guidance, navigation and control conference (CGNCC2012), Beijing, 2012. (in Chinese)

11. Lawrence C, Mills B. Status update of the AEDC virtual flight testing development program. AIAA 2002-0168, 2002.

12. Magill J C, Cataldi P, Morency J R, Hammer D X, Burgess R, Jeter E. Demonstration of a wire suspension for wind-tunnel virtual flight testing. Journal of Spacecraft and Rockets, 2009, 46(3): 624-633.

13. Huang $M$, Wang $Z$ W. A review of wind tunnel based virtual flight testing techniques for evaluation of flight control systems. International Journal of Aerospace Engineering, 2015(1), 1 22.

14. Huang M, Wang Z W, Guo Z Y, Niu Y B. Design of the wind tunnel based virtual flight testing evaluation method for flight control systems. Proc IMechE Part G: Journal of Aerospace Engineering, 2016, 0(0): 1-13.

15. Huang $M$, Wang $Z$ W, Guo Z Y. Wind tunnel based virtual flight testing system and key technologies for the evaluation of flight control system. Journal of National University of Defense Technology, 2017, 39(2): 1-8. (in Chinese)

16. Huang M, Wang Z W, Guo Z Y, Zeng Q H, Niu Y B. Wind tunnel hardware-in-the-loop simulation techniques for flight control system evaluation. 2017 2nd International Conference on Control, Automation and Artificial Intelligence, 2017.

17. Zeng Q H, Guo Z Y. Unmanned flight control techniques and engineering. Beijing: National Defense Industry Press, 2011. (in Chinese)

18. Gao J Y, Li L Y, Feng Y C, etc. Aircraft flight qualities. Beijing: National Defense Industry Press, 2011. (in Chinese) 